日本原子力研究開発機構機関リポジトリ

Japan Atomic Energy Agency Institutional Repository

\begin{tabular}{|c|l|}
\hline Title & $\begin{array}{l}\text { Structures of quasi-freestanding ultra-thin silicon films deposited } \\
\text { on chemically inert surfaces }\end{array}$ \\
\hline Author(s) & Baba Yuji, Shimoyama Iwao, Hirao Norie, Sekiguchi Tetsuhiro \\
\hline Citation & Chemical Physics, 444,p.1-6 \\
\hline Text Version & Author's Post-print \\
\hline URL & $\underline{\text { https://jopss.jaea.go.jp/search/servlet/search?5048549 }}$ \\
\hline DOI & $\underline{\text { https://doi.org/10.1016/j.chemphys.2014.09.011 }}$ \\
\hline Right & $\begin{array}{l}\text { C } 2014 . \text { This manuscript version is made available under the } \\
\text { CC-BY-NC-ND } 4.0 \text { license } \\
\text { http://creativecommons.org/licenses/by-nc-nd/4.0/ }\end{array}$ \\
\hline
\end{tabular}




\title{
Structures of quasi-freestanding ultra-thin silicon films deposited on chemically inert surfaces
}

\author{
Y. Baba ${ }^{*}$ I. Shimoyama, N. Hirao and T. Sekiguchi \\ Japan Atomic Energy Agency, Tokai-mura, Naka-gun, Ibaraki, 319-1195, Japan
}

\begin{abstract}
Silicon thin films were deposited on a sapphire and a highly oriented pyrolytic graphite (HOPG), which have atomically flat and chemically inert surfaces. The electronic and geometrical structures of the films were analyzed by X-ray photoelectron spectroscopy (XPS) and polarization-dependent X-ray absorption fine structure (XAFS). It was found that the silicon $K$-edge XAFS spectra for ultra-thin silicon films thinner than 0.2 monolayer exhibited two distinct resonance peaks which were not observed for bulk silicon. The peaks were assigned to the resonance excitations from the $\mathrm{Si} 1 s$ into the valence unoccupied orbitals with $\pi^{*}$ and $\sigma^{*}$ characters. The average tilted angle of the $\pi^{*}$ orbitals was determined by the polarization dependencies of the peak intensities. It was demonstrated that direction of a part of the $\pi^{*}$ orbitals in silicon film is perpendicular to the surface. These results support the existence of quasi-freestanding single-layered silicon films with $s p^{2}$ configuration.
\end{abstract}

*Corresponding author

Present address: Sector of Fukushima Research and Development, Japan Atomic Energy Agency, 6-6, Sakae-machi, Fukushima-shi, Fukushima 960-8031, Japan e-mail: baba.yuji@jaea.go.jp

Keywords: silicon thin film; sapphire; HOPG; XAFS; polarization dependence; synchrotron radiation 


\section{Introduction}

Graphene, one of the carbon allotropes, has recently attracted considerable attention because of its excellent optical and electrical properties. Graphene is atomically flat single-layered sheet exhibiting honeycomb structure containing $s p^{2}$ configuration. Silicon belongs to the same group as carbon in the periodic table, and generally takes tetra-valence states like carbon. However in contrast to carbon, silicon tends to form $s p^{3}$ configuration with the surrounding silicon atoms in solid phase. Even for a thin film, silicon atoms deposited on a solid surface generally agglomerate, and form three-dimensionally spread diamond-like structure with $s p^{3}$ bonds.

In spite of these generally accepted features, recent theoretical works have shown that there exists a stable phase of single-layered silicon with graphene-like structure either in a flat or in a slightly puckered configuration [1-10]. A single-layered silicon with graphene-like structure is recently called "silicene". If silicene structure surely exists, an outstanding characteristic is the presence of a Dirac cone in the band structure like graphene [3]. The existence of a Dirac cone results in the gapless excitations with a linear dispersion, which leads to the applications to optoelectronics and transistors in micro-electronics composed of only silicon atoms. Furthermore a graphene-like silicon may exhibit excellent electric properties such as tunable band-gap induced by quantum spin Hall effect and topological modification [11-21]. However, it should be noted that the graphene-like silicon dealt with in these theoretical works are assumed to be "freestanding", where the interaction between the substrate and silicon is not fully considered.

Up to now, many experimental works have been conducted as to the synthesis of silicene [22-36]. In these works, metallic single crystals such as $\operatorname{Ag}(111)$ were used as substrates. However, it should be noted that silicon atoms are highly reactive with many metals and tend to form stable silicide. Therefore the contribution of the hybridization between silicon and substrate cannot be excluded in these experimental works. Actually it was reported that band structure of silicene deposited on $\operatorname{Ag}(111)$ drastically modified by the hybridization between the silicon and silver atoms, and the silicene loses its Dirac fermion characteristics due to substrate-induced symmetry breaking [37]. The effect of the hybridization between silicene and metals was supported by the theoretical 
calculations $[38,39]$.

As to the graphene-like silicon that is weakly bound on the substrate, Fleurence et al. have recently showed that graphene-like silicon is formed through surface segregation on zirconium diboride thin films grown on $\mathrm{Si}$ wafers [40]. The layer obtained in their work was buckled. They concluded that the electronic properties of single-layered silicon are modified by the buckling due to epitaxial strain. Neek-Amal et al. have calculated by density functional theory that singe-layered silicon intercalated between graphene layers is weakly bound though van der Waals force, and it is possibly stable well above the room temperature [41]. The stable silicon layer calculated by them was also buckled hexagonal arrangement. In spite of these works, a question still remains as to whether a "freestanding" sheet of mono-layered silicon with graphene-like structure surely exists or not.

As mentioned above, silicon atoms tend to interact with most of metals forming stable silicide, so the better way to synthesize "freestanding" graphene-like silicon is to deposit silicon on "atomically flat" and "chemically inert" surfaces. In the previous communication, we presented the results for the X-ray absorption fine structure (XAFS) analysis for ultra-thin silicon films deposited on a highly oriented pyrolytic graphite (HOPG) [42]. On the basis of qualitative speculation, we have shown that a part of the deposited layer lies flat on the surface [42].

In the present paper, we show the XAFS spectral data for ultra-thin films of silicon deposited on sapphire substrate as well as on HOPG. Both substrates are chemically inert and atomically flat, so it is appropriate surfaces for the deposition of freestanding silicon films. Transparent and insulating properties of sapphire are suited for future applications of silicene as optical and electric devices. On the basis of the quantitative analyses of the polarization dependencies in the Si $K$-edge XAFS spectra, the average tilted angle of the molecular orbitals relative to the surface was determined, and the existence of quasi-freestanding graphene-like silicon film is discussed.

\section{Experimental}

As substrates, a sapphire (C-plane (0001), $10 \mathrm{~mm} \times 10 \mathrm{~mm} \times 0.2 \mathrm{mmt}$, Jiaozuo City Crystal Photoelectric Materials Co., Ltd.) and an HOPG (10 mm×10 mm×1 mmt, 
Techno Chemics Inc.) were used. The surface of the sapphire was polished, and it was annealed at $900^{\circ} \mathrm{C}$ for $60 \mathrm{~min}$ in air to prepare an atomically flat surface. The sapphire was then rinsed by supersonic waves in ethanol for $15 \mathrm{~min}$, and was introduced into the vacuum chamber. A fresh surface of HOPG was obtained by cleaving in dry nitrogen atmosphere. Then the sample was transferred into the vacuum chamber without exposing to air.

For the evaporation of silicon, a high-purity silicon rod was used as a source material. The evaporator consisted of a tantalum crucible surrounded by the spiral tungsten filament. The crucible was floated at $+1.5 \mathrm{kV}$. The filament was grounded, so the crucible was heated by the bombardment of $1.5 \mathrm{keV}$ electrons. The distance between the crucible and the substrate was $20 \mathrm{~mm}$. A shutter that is electrically isolated from the ground was set between the crucible and the substrate. A part of evaporated silicon atoms was ionized due to the surface ionization, thus a positive current was observed at the shutter during the evaporation. The thickness of the film was estimated by the product of the shutter current and the evaporation time using a calibration curve obtained in advance by the X-ray photoelectron spectra (XPS). The base pressure of evaporation chamber was $5 \times 10^{-9} \mathrm{~Pa}$, and the typical vacuum pressure during the deposition was $1.3 \times 10^{-4} \mathrm{~Pa}$. The substrate was kept at room temperature during the evaporation.

The XPS and XAFS measurements were performed at the soft X-ray beamline (BL-27A) of the Photon Factory in the High Energy Accelerator Research Organization (KEK-PF). In this beamline, photons were emitted from the bending magnet, and they were linearly polarized at the horizontal direction. The photon energy was tuned by an $\mathrm{InSb}(111)$ double crystal monochromator. The typical photon flux was $\sim 10^{10}$ photons $\mathrm{cm}^{-2} \cdot \mathrm{s}^{-1}$. The energy resolution of the monochromator was $0.85 \mathrm{eV}$ at $1850 \mathrm{eV}$ (around Si $K$-edge).

The experimental apparatus at the end of the beamline was composed of two vacuum chambers, i.e., analysis chamber and preparation chamber. The analysis chamber consisted of a four-axis manipulator, an electron energy analyzer, and an electron flood gun for surface charge compensation. The base pressure of the analysis chamber was $1 \times 10^{-8} \mathrm{~Pa}$. The sample was vertically located, and it was rotated around the vertical axis. The preparation chamber consisted of a vacuum evaporator and a sample transfer 
system. The base pressure of the preparation chamber was $5 \times 10^{-9} \mathrm{~Pa}$. The sample can be transferred between two chambers without exposing to air.

XPS spectra were measured with hemispherical electron energy analyzer (VSW Co. Class-100). The X-rays were irradiated at $35^{\circ}$ from the surface, and the take-off direction of photoelectrons was surface normal. Typical photon energy used in XPS measurements was $3000 \mathrm{eV}$. Sapphire is a wide-gap insulator, so the surface charging induced by photoemission caused a serious problem in XPS measurements. Using electron flood gun, the charging effect was fairly reduced, and the kinetic energy of all photoelectrons were linearly shifted by a few eV to lower energy. So, the binding energy for sapphire substrate was calibrated by the $\mathrm{O} 1 \mathrm{~s}$ photoelectrons from sapphire at 531.6 $\mathrm{eV}$ [43]. For HOPG, the binding energy was calibrated by the $\mathrm{C} 1 s$ photoelectrons from HOPG at $284.3 \mathrm{eV}$ [43].

The XAFS spectra were measured by plotting a sample drain current as a function of the photon energy (total electron yield, TEY). The sample current was normalized by the photon flux measured by the drain current of an aluminum mesh located in front of the sample. Before the silicon deposition, we measured the XAFS spectra for clean surfaces of sapphire and HOPG as background spectra. These background spectra were subtracted from the XAFS spectra after the silicon deposition.

\section{Results and discussion}

Figure 1 shows the Si $1 s$ XP spectra of silicon deposited on sapphire (left) and HOPG (right). The thickness of the silicon layer was estimated from the XPS peak intensity ratio of silicon ( $\mathrm{Si} 1 s$ ) to substrate (Al $1 s$ for sapphire and $\mathrm{C} 1 s$ for HOPG). In these estimation, we assumed that the deposited layer is homogeneous. So the thickness and number of silicon layers shown in the figure are averaged values. The details of the thickness calculation were described elsewhere [42]. For sapphire (left figure), the energy of the Si $1 s$ peak (marked A) for 5.5-layered sample is located at $1840.9 \mathrm{eV}$. This energy is almost the same as that for bulk silicon [44]. Also a plasmon loss peak (marked B) is clearly seen at $18 \mathrm{eV}$ higher binding energy (lower kinetic energy). Thus, 5.5-layered sample exhibits bulk feature. On the other hand, the peak energy for the 0.09 -layered film (marked C) is higher by $4.5 \mathrm{eV}$ than that for the 5.5-layered film. The 
higher binding energy peak for a thin film than that for the bulk are not due to the formation of $\mathrm{SiO}_{2}$ as following reasons. Firstly, evaporation was conducted under ultra-high vacuum condition without oxygen contamination. Secondly, if the deposited silicon is bonded to oxygen in sapphire, the silicon atom would be bonded to at most two oxygen atoms. Thus silicon atoms do not form $\mathrm{SiO}_{4}$ network but partially oxidized. The present energy shift $(4.5 \mathrm{eV})$ is larger than that for partially oxidized silicon such as divalent silicon [45]. Furthermore, the Si $K$-edge XAFS spectra for the thin films is different from that for $\mathrm{SiO}_{2}$ (see Fig. 2, discussed later). This fact also confirms that the higher binding energy of the $\mathrm{Si} 1 s$ for 0.09 layer is not due to the formation of $\mathrm{SiO}_{2}$. Such energy difference between thick and ultra-thin films is also observed for HOPG substrate (right figure). The energy of Si $1 s$ peak observed for 1.8 layer and 0.15 layer (marked F) is higher by $4.2 \mathrm{eV}$ than that for bulk 38-layered sample (marked D). This energy shift is also not due to the formation of silicon oxide, because $\mathrm{O} 1 \mathrm{~s}$ peak was not observed in XP spectra.

The higher binding-energy shift observed for ultra-thin films is interpreted as follows. The core-level energy in XPS is measured from the Fermi level. Thus the core-level energy shift represents the changes in the ionization potential of the solid surface. It is generally accepted that the ionization potentials of low-dimensional materials such as clusters, nano-wires and nano-dots are higher than those of the corresponding bulk materials. For low-dimensional silicon, it was reported that the ionization potential of silicon is size-dependent $[46,47]$. According to these reports, the ionization potential of silicon decreases with the cluster size, and the value saturates around $\mathrm{Si}_{40}$. The difference in the ionization potential between silicon monomer and $\mathrm{Si}_{\mathrm{n}}$ $(\mathrm{n}>40)$ is about $4 \mathrm{eV}$, which is close to the present energy difference between thick and thin films. For XPS of silicon thin film, Nath et al. have found that the binding energy of the Si 1s peak for thin film was thickness-dependent, and the peak energy for Si thin film is a few eV higher than that for bulk silicon [48]. Such higher-energy shifts of XPS peak for thin films were also reported for the other systems such as adsorbed molecules [49], and deposited metals [50]. Thus the present results for the Si $1 s$ energy shift between thick and thin films is interpreted by the changes in the ionization potentials depending on the film thickness. If the deposited silicon atoms agglomerate and form 3D islands, such higher binding-energy shift would be scarcely observed. Thus the 
present results confirm that low-dimensional silicon is the major part in the deposited film.

Figure 2 shows the Si $K$-edge XAFS spectra for silicon deposited on sapphire (c) and HOPG (d). For comparison, XAFS spectra for bulk $\mathrm{SiO}_{2}$ and $\mathrm{Si}(001)$ single crystal are also displayed in (a) and (b), respectively. For bulk $\operatorname{Si}(001)$, the main peak at 1842 $\mathrm{eV}$ (marked (1)) is due to the resonance excitation from the $\mathrm{Si} 1 s$ into $\sigma^{*}$ orbitals with $\mathrm{Si}$ $3 p$ character [51]. For 5.5-layered silicon on sapphire (c) and 4.1-layered silicon on HOPG (d), the intensity of the peak (1) decreases. This bulk resonance peak disappeared for further thin films (see Fig. 3, explained later). Instead, two intense peaks (marked (2) and (3)) appear at higher energy region. These peaks are not due to the formation of $\mathrm{SiO}_{2}$, because the XAFS spectrum of $\mathrm{SiO}_{2}$ has a single sharp resonance peak at higher energy, as seen in (a). The peaks (2) and (3) are attributed to the resonance excitations from the $\mathrm{Si} 1 s$ to the valence unoccupied states in low-dimensional silicon film.

The spectral feature of XAFS is a little different from that of XPS. For sapphire substrate, Si $1 s$ XPS spectrum for 5.5-layered sample exhibits only bulk feature (Fig. 1, left). While the XAFS spectrum represents the convolution of bulk and low-dimensional spectra (Fig.2 (c)). This discrepancy is interpreted by the difference in the detection depth between XPS and XAFS. For XPS, the detection depth of Si $1 s$ photoelectron is about $1 \mathrm{~nm}$. While XAFS in total electron yield mode probes in the order of $10 \mathrm{~nm}$, because most of the electrons emitted form surface have low kinetic energy, whose inelastic mean free path (IMFP) is fairly larger than that of Si $1 s$ photoelectron. So the difference between XPS and XAFS suggests that for 5.5-layered sample the first silicon layer on sapphire has low-dimensional character and the upper layer exhibits bulk feature.

Deduced from the C $K$-edge XAFS spectra for graphite [52], it is suggested that the higher-energy peaks in XAFS (Fig.2, peaks (2) and (3)) are due to the resonance excitations from the Si $1 s$ into the valence unoccupied states with $\pi^{*}$ and $\sigma^{*}$ characters. However, it has not become clear whether or not a single-layered silicon has really $\pi$ bonds like graphite. As to this point, Padova et al. have measured Si $K$-edge reflection electron energy loss spectra (REELS) as a function of the electron beam incidence angle 
for silicon nano-ribbons grown on $\operatorname{Ag}(110)$ [53]. They have found the two distinct loss structures attributed to the Si $1 s \rightarrow \pi *$ and $\mathrm{Si} 1 s \rightarrow \sigma *$ transitions on the basis of peak intensity dependence on incidence angle. They concluded that the $s p^{2}$-like hybridization of the silicon valence orbitals exists in the silicon nano-ribbons. Although the $\mathrm{Si} 1 s \rightarrow \pi *$ and Si $1 s \rightarrow \sigma *$ transition peaks were not separated due to the poor energy resolution of REELS, this is the first result that identify the $\pi$ bond in low-dimensional silicon. The present results are in principle similar to their results, but XAFS spectra have fairly better energy resolution than that of REELS. So two peaks are clearly separated.

In order to confirm the peak assignments and energy of the two resonance peaks in XAFS, we have calculated the electronic structures of mono-layered silicon with graphene-like structure using discrete variational (DV)-X $\alpha$ method. In this calculation, we assumed that the mono-layered silicon is flat like graphene. As a result, tt was elucidated that the valence unoccupied orbitals are composed of two distinct states. The lower energy state is attributed to out-of-plane molecular orbital that consists of mainly Si $3 p$ orbitals, and the components of the higher energy state are attributed to in-plane molecular orbitals [54]. On the basis of this calculation and the reported experimental results by REELS [53], we assign that the peak (2) in Fig. 2 corresponds to the resonance from the $\mathrm{Si} 1 s$ into $\mathrm{Si} 3 p_{\mathrm{z}}{ }^{*}$ that is perpendicular to the surface (out-of-plane, hereafter indicated as Si $1 s \rightarrow \pi^{*}$ ), and the peak (3) is due to that from Si $1 s$ into the orbitals that are parallel to the molecular plane (in-plane, hereafter indicated as $\mathrm{Si}$ $\left.1 s \rightarrow \sigma^{*}\right)$.

Figure 3 shows the Si $K$-edge XAFS spectra for the thinner silicon film deposited on sapphire in an expanded energy region as compared with Fig. 2. The thickness of the layer is 0.10 layer which is fairly thinner than that for Fig. 2(c). The spectra for two different incident angles $\left(20^{\circ}\right.$ and $\left.80^{\circ}\right)$ are shown. The bulk peak around $1841 \mathrm{eV}$ completely disappeared, and only Si $1 s \rightarrow \pi^{*}$ peak (marked (a)) and Si $1 s \rightarrow \sigma^{*}$ peak (marked (b)) are observed. The intensity of the two spectra was normalized by the intensity at $1870 \mathrm{eV}$ where the angle dependence was not observed. An incident-angle dependence is observed for the intensity of the peak (a). The intensity of the peak (a) at $20^{\circ}$ incidence is enhanced as compared with that at $80^{\circ}$ incidence. Since the synchrotron $\mathrm{X}$-rays from the bending magnet is linearly polarized at horizontal direction, the 
incident-angle dependence of the peak intensity suggests that the final-state orbitals represented by the peak (a) points to certain directions.

First, we will qualitatively estimate the direction of the orbitals. A peak intensity $I$ in the XAFS spectra using the synchrotron beam of electric field vector $\boldsymbol{E}$ is expressed as,

$I \propto|\boldsymbol{E} \cdot \boldsymbol{O}|^{2} \propto \cos ^{2} \delta$

where $\boldsymbol{O}$ is the vector of the final state orbital and $\delta$ is the angle between $\boldsymbol{E}$ and $\boldsymbol{O}$ [55]. As described above, the final-state orbitals of the resonance represented by the peak (a) has $\pi^{*}$ character (out-of-plane), so it is deduced that $\boldsymbol{O}$ points to perpendicular to the surface. This is really the case for graphite where the final state orbitals $\left(\pi^{*}\right)$ consist of $\mathrm{C}$ $2 p_{\mathrm{z}}{ }^{*}[52]$.

Next, we will analyze the orientation of the orbitals more quantitatively. Fig. 4 presents the incident angle dependence of the Si K-edge XAFS spectra for ultra-thin silicon film deposited on HOPG. The thickness of the layer is 0.15 layer, which is thin enough, so the bulk peak is not observed. Similarly to the sapphire case, the Si $1 s \rightarrow \pi^{*}$ resonance peak (marked (a)) and Si $1 s \rightarrow \sigma^{*}$ peak (marked (b)) are observed. A clear incident angle dependence of the peak intensity is observed. The intensity of the peak (a) relative to that of the peak (b) decreases with the incident angle.

First, the precise intensity of Si $1 s \rightarrow \pi^{*}$ peak was obtained by a peak deconvolution using Gaussian-curve fitting. The example for the curve fitting of the XAFS spectrum at $\theta=40^{\circ}$ is shown in the left side of Fig. 5. The resonance peak intensity in the XAFS spectrum is given as

$$
I(\theta)=A \cdot\left[P \cdot I_{P}+(1-P) \cdot I_{V}\right]
$$

where $I_{P}$ and $I_{V}$ are the transition intensities associated with the angle-dependent matrix elements parallel and vertical, respectively, to the electric vector, $\theta$ is the incidence angle of the synchrotron beam, $A$ is the normalization factor, and $P$ is the polarization factor. If we assume graphene-like structure, the layer has a symmetry higher than threefold. In this case, the intensities are simply expressed as 


$$
I_{P}=(1 / 3) \cdot\left[1+(1 / 2) \cdot\left(3 \cos ^{2} \theta-1\right) \cdot\left(3 \cos ^{2} \alpha-1\right)\right]
$$

and

$$
I_{V}=(1 / 2) \cdot \sin ^{2} \alpha
$$

where $\alpha$ is the polar angle of the final state orbitals [55]. The polarization factor of the synchrotron beam in the present experimental condition is estimated to be about 0.95 [56]. The theoretically calculated values of $I$ for various $\alpha$-values are plotted as a function of the incident angle $\theta$. The results are shown as solid lines in the right side of Fig. 5. The vertical scale is normalized such that the intensity at $\theta=54.7^{\circ}$ (magic angle) are $1 / 3$. The normalized intensity of the $\mathrm{Si} 1 s \rightarrow \pi^{*}$ peak (a) obtained from the curve fitting is shown as filled circles. The intensities of the peak (a) were normalized by the intensity at $1870 \mathrm{eV}$ for respective incident angles where no polarization dependence exists. The values were further normalized such that the intensity at $\theta=54.7^{\circ}$ are $1 / 3$. At first glance, the experimental data follow the line of $\alpha=40^{\circ}$. This means that the averaged polar angle of the $\pi^{*}$ orbitals is $40^{\circ}$. In the case of graphene, the molecular plane and $\pi^{*}$ orbitals are orthogonal, so the result indicate that the molecular plane tilted at $40^{\circ}$ from the surface.

If the average tilted angle of single-layered silicon is $40^{\circ}$, two cases are possible. In the first case, all of the films are tiled at about $40^{\circ}$. However, the surface of HOPG is atomically flat, so there is no reason that all of the layers are tilted at the same angles. In the second case, a part of the layer lies flat on the surface $\left(\alpha=0^{\circ}\right)$ and the remaining part is randomly oriented $\left(\alpha=54.7^{\circ}\right)$. At present we cannot prove that the second case is correct, because we have not yet measured nano-structures of the film at atomic scale. But we suppose that a part of the silicon film lies flat by a simple speculation as follows.

It has been reported that ultra-thin silicon films deposited on HOPG [57] and glass [58] substrates agglomerate with three-dimensional (3D) growth mode (Volmer-Weber growth mode $[59,60])$ at room temperature on the basis of the observation by scanning probe microscope. The present experimental condition is similar to those in these reports. Thus the 3D structure with random orientation $\left(\alpha=54.7^{\circ}\right)$ is surely included in the deposited film. However, the thickness of the silicon film shown in Figs. 3 and 4 is 
fairy thinner than those reported in [57,58]. So, at the initial stage of the deposition, silicon would be deposited with another growth mode exhibiting the polarization dependence shown in Figs.3 and 4. If a part of $x$ in the film has a certain orientation, i.e., the polar angle of the unoccupied orbitals corresponding to $\alpha$ (shown in equation (4)), and the remaining part is randomly oriented, the following equation holds,

$$
\alpha \cdot x+54.7 \cdot(1-x)=40
$$

When 0 is substituted in $\alpha$, we obtain $x=0.27$. Thus it is simply estimated that about $27 \%$ of the unoccupied $\pi^{*}$ orbitals of silicon layer points to perpendicular to the surface, and remaining $73 \%$ is randomly oriented. The present results do not fully prove the existence of a stable phase of single-layered silicon with graphene-like structure. Also we cannot conclude whether the layer has perfectly flat or slightly puckered configuration, because the molecular orbitals are hybridized. But we stress that the present result supports the existence of quasi-freestanding monolayered silicon with $\pi^{*}$ orbitals, pointing to perpendicular to the surface. In the present work, we showed the averaged structure (in the order of $1 \mathrm{~mm}^{2}$ ) for the quasi-freestanding ultra-thin silicon film based on the spectrometric methods using X-rays. The observation of nano-structure at atomic scale would be indispensable as a next step in order to further confirm the existence of single-layered silicon with graphene-like structure.

\section{Summary}

Ultra-thin films of silicon was deposited on chemically inert surfaces; sapphire and HOPG, in order to synthesize quasi-freestanding single-layered silicon. The structures of silicon films were analyzed by XPS and Si $K$-edge XAFS using linearly polarized synchrotron radiation. It was found that the Si $K$-edge XAFS spectra for ultra-thin silicon films less than 0.2 monolayer exhibits two distinct resonance peaks. These peaks correspond to the excitations from the Si $1 s$ into the valence unoccupied orbitals with $\pi^{*}$ and $\sigma^{*}$ characters. On the basis of the analyses of the polarization dependencies of the XAFS spectra, it was demonstrated that about $27 \%$ of the unoccupied $\pi^{*}$ orbitals of 
silicon layer points to perpendicular to the surface. The results support the existence of quasi-freestanding ultra-thin silicon films with $s p^{2}$ configuration.

\section{Acknowledgements}

The authors would like to thank the staff of the KEK-PF for their assistance throughout the experiments. The work has been conducted under the approval of Photon Factory Program Advisory Committee (PF-PAC 2012G553).

\section{References}

[1] K. Takeda, K. Shiraishi, Phys. Rev. B 50 (1994) 14916.

[2] G.G. Guzmán-Verri, L.C. Lew yan Voon, Phys. Rev. B 76 (2007) 075131.

[3] S. Cahangirov, M. Topsakal, E. Akturk, H. Sahin, S. Ciraci, Phys. Rev. Lett. 102 (2009) 236804.

[4] M. Topsakal, S. Ciraci, Phys. Rev. B 81 (2010) 024107.

[5] M. Houssa, G. Poutois, V.V. Afanas'ev, A. Stesmans, Appl. Phys. Lett. 97 (2010) 112106.

[6] B. Mohan, A. Kumar, P.K. Ahluwalia, Physica E: Low-Dimensional System Nanostrtucture 53 (2013) 233.

[7] S. Kokott, P. Pflugradt, L. Matthes, F. Bechstedt, J. Phys.: Condens. Matter. 26 (2014) 185002.

[8] C. Kamal, A. Chakrabarti, A, Banerjee, Phys. Lett. A 378 (2014) 1162.

[9] S. Trivedi, A. Srivastava, R. Kurchania, J. Comput. Theor. Nanosci. 11 (2014) 781.

[10] D. Jose, A. Datta, Acc. Chem. Res. 47 (2014) 593.

[11] C.C. Liu, W. Feng, Y. Yao, Phys. Rev. Lett. 107 (2011) 076802.

[12] M. Ezawa, J. Phys. Soc. Jpn. 81 (2012) 064705.

[13] M. Ezawa, Phys. Rev. Lett. 109 (2012) 055502.

[14] A. Dyrdal, J. Barnas, Phys. Stat. Sol. Rapid Res. Lett. 6 (2012) 340.

[15] M. Tahir, U. Schwingenschlogl, Sci. Rep. 3 (2013) 1075.

[16] X.T. An, Y.Y. Zhang, J.J. Liu, S.S. Li, Appl. Phys. Lett. 102 (2013) 043113. 
[17] M. Ezawa, Phys. Rev. B 87 (2013) 155415.

[18] M. Tahir, A. Manchon, K. Sabeeh, U. Schwingenschlogl, Appl. Phys. Lett. 102 (2013) 162412.

[19] F. Geissler, J.C. Budich, B. Trauzettel, New J. Phys. 15 (2013) 085030.

[20] Y. Kim, K.Choi, J. Ihm, H. Jin, Phys. Rev. B 89 (2014) 085429.

[21] H. Pan, Z. Li, C.C. Liu, G. Zhu, Z. Qiao, Y. Yao, Phys. Rev. Lett. 112 (2014) 106802.

[22] B. Aufray, A. Kara, S. Vizzini, H. Oughaddou, C. Léandri, B. Ealet, G. Le Lay. Appl. Phys. Lett. 96 (2010) 183102.

[23] C.L. Lin, R. Arafune, K. Kawahara, N. Tsukahara, E. Minamitani, Y. Kim, N. Takagi, M. Kawai, Appl. Phys. Express 5 (2012) 045802.

[24] H. Jamgotchian, Y. Colignon, N. Hamzaoui, B Ealet, J.Y. Hoarau, B. Aufray, J.P. Bibérian, J. Phys.: Condens. Matter 24 (2012) 172001.

[25] B. Feng, Z. Ding, S. Meng, Y. Yao, X. He, P. Cheng, L. Chen, K. Wu, Nano Lett. 12 (2012) 3507.

[26] J. Gao, J. Zhao, Sci. Rep. 2 (2012) 861.

[27] R. Arafune, C.L. Lin, K. Kawahara, N. Tsukahara, E. Minamitani, Y. Kim, N. Takagi, M. Kawai, Surf. Sci. 608 (2013) 297.

[28] Z. Majzik, M.R. Tchalala, M. Svec, P. Hapala, H. Enriquez, A. Kara, A.J. Mayne, G. Dujardin, P. Jelínek, H. Oughaddou, J. Phys.: Condens. Matter 25 (2013) 225301.

[29] S. Cahangirov, M. Audiffred, P. Tang, A. Iacomino, W. Duan, G. Merino, A. Rubio, Phys. Rev. B 88 (2013) 035432.

[30] A. Resta, T. Leoni, C. Barth, A. Ranguis, C. Becker, T. Bruhn, P. Vogt, G.L. Lay, Sci. Rep. 3 (2013) 2399.

[31] Y. Fukaya, I. Mochizuki, M. Maekawa, K. Wada, T. Hyodo, I. Matsuda, A. Kawasuso Phys. Rev. B 88 (2013) 205413.

[32] D. Tsoutsou, E. Xenogiannopoulou, E. Golias, P. Tsipas, A. Dimoulas, Appl. Phys. Lett. 103 (2013) 231604.

[33] Y. Yuan, R. Quhe, J. Zheng, Y. Wang, Z. Ni, J. Shi, J. Lu, Physica E: Low-dimensional System Nanostructure 58 (2014) 38.

[34] K. Kawahara, T. Shirasawa, R. Arafune, C.L. Lin, T. Takahashi, M. Kawai, N. Takagi, Surf. Sci. 623 (2014) 25. 
[35] P. Moras, T.O. Mentes, P.M. Sheverdyaeva, A. Locatelli, C. Carbone, J. Phys.: Condens. Matter 26 (2014) 185001.

[36] M. Tchalala, R. Mohamed, H. Enriquez, H. Yildirim, A. Kara, A.J. Mayne, G. Dujardin, M.A. Ali, H. Oughaddou, Appl. Surf. Sci. 303 (2014) 61.

[37] C.L. Lin, R. Arafune, K. Kawahara, M. Kanno, N. Tsukahara, E. Minamitani, Y. Kim, M. Kawai, N. Takagi, Phys. Rev. Lett. 110 (2013) 076801.

[38] Z.X. Guo, S. Furuya, J. Iwata, A. Oshiyama, Phys. Rev. B 87 (2013) 235435.

[39] Y.P. Wang. H.P. Cheng, Phys. Rev. B 87 (2013) 245430.

[40] A. Fleurence, R. Friedlein, T. Ozaki, H. Kawai, Y. Wang, Y. Yamada-Takamura, Phys. Rev. Lett. 108 (2012) 245501.

[41] M. Neek-Amal, A. Sadeghi, G.R. Berdiyorov, F.M. Peeters, Appl. Phys. Lett. 103 (2013) 261904.

[42] Y. Baba, I Shimoyama, N. Hirao, T. Sekiguchi, Chem. Phys. Lett. 594 (2014) 64.

[43] C.D. Wagner, W.M. Riggs, L.E. Davis, J.F. Moulder, G.E. Mullenberg, "Handbook of X-ray photoelectron spectroscopy”, Perking-Elmer Corp., MN, USA, 1979.

[44] Y. Baba, T. Sekiguchi, I. Shimoyama, K.G. Nath, Appl. Surf. Sci. 234 (2004) 246.

[45] Y. Baba, T. Sekiguchi, I. Shimoyama, N. Hirao, Surf. Sci. 612 (2013) 77.

[46] K. Fuke, K. Tsukamoto, F. Misaizu, M. Sanekata, J. Chem. Phys. 99 (1993) 7807.

[47] N. Binggeli, J. R. Chelikowasky, Phys. Rev. Lett. 75 (1995) 493.

[48] Krishna G. Nath, I. Shimoyama, T. Sekiguchi, Y. Baba, J. Appl. Phys. 94 (2003) 4583.

[49] M.J. Breitschafter, E. Umbach, D. Menzel, Surf. Sci. 178 (1986) 725.

[50] K. Kishi, Y. Hayakawa, K. Fujiwara, Surf. Sci. 356 (1996) 171.

[51] A.P. Hitchcock, T. Tyliszczak, P. Aebi, J.Z. Xiong, T.K. Sham, K.M. Baines, K.A. Mueller, X.H. Feng, J.M. Chen, B.X. Yang, Z.H. Lu, J.-M. Baribeau, T.E. Jackman, Surf. Sci. 291 (1993) 349.

[52] R.A. Rosenberg, P. Love, P. R. LaRoe, V. Rehn, C. C. Parks, Phys. Rev. B 31 (1985) 2634.

[53] P. De Padova, C. Quaresima, B. Olivieri, P. Perfetti, G. Le Lay, Appl. Phys. Lett. 98 (2011) 081909.

[54] I. Shimoyama, private communication.

[55] J. Stöhr, "NEXAFS spectroscopy," Springer Series in Surface Sciences, Vol. 25, 
Springer, Berlin New York, 1996.

[56] H. Kitamura, Synchrotron Radiation Calculation Program for PC98, V.2.3 (1993).

[57] P. Scheier, B. Marsen, M. Lonfat, W. D. Schneider, K. Sattler, Surf. Sci. 458 (2000) 113.

[58] D. Li, G.L. Liu, Y. Yang, J.H. Wu, Z.R. Huang, J. Nanomaterials 2013 (2013) 383867.

[59] E. Bauer, Z. Kristallogr. 110 (1958) 372.

[60] E. Bauer, Appl. Surf. Sci. 11-12 (1982) 479. 


\section{Figure captions}

Figure 1. Narrow-scan XPS spectra in Si $1 s$ region for silicon deposited on sapphire (left figure) and $\mathrm{Si}(001)$ (right figure). Photon energy used was $3000 \mathrm{eV}$. Thickness of the silicon layer is indicated in each spectrum. The thickness of the silicon layer was estimated from the XPS peak intensity ratio of silicon ( $\mathrm{Si} 1 s$ ) to substrate (Al $1 s$ for sapphire and $\mathrm{C} 1 s$ for HOPG) assuming that the deposited layer is homogeneous.

Figure 2. $\mathrm{Si} K$-edge XAFS spectra for (a) $\mathrm{SiO}_{2}$, (b) $\mathrm{Si}(001)$, (c) $\mathrm{Si}$ deposited on sapphire, and (d) Si deposited on HOPG. Thickness of the silicon layer is indicated in each spectrum.

Figure 3. Si $K$-edge XAFS spectra measured by two X-ray incident angles for Si deposited on sapphire. The thickness of the layer was 0.10 monolayer.

Figure 4. Si $K$-edge XAFS spectra measured by various X-ray incident angles for Si deposited on HOPG. The thickness of the layer was 0.15 monolayer.

Figure 5. Left: An example for the curve fitting of the Si $K$-edge XAFS spectrum at $\theta=40^{\circ}$ for silicon on HOPG. Right: Calculated normalized intensity of the $\mathrm{Si}$ $1 s \rightarrow \pi^{*}$ resonance peak in the Si $K$-edge XAFS spectra as a function of the $\mathrm{X}$-ray incidence angle for various $\alpha$ values (solid lines). The vertical scale is normalized such that the intensity at $\theta=54.7^{\circ}$ (magic angle) are $1 / 3$. The normalized intensity of the Si $1 s \rightarrow \pi^{*}$ peak (a) obtained from the curve fitting is shown as filled circles. The intensities of the peaks (a) were normalized by the intensity at $1870 \mathrm{eV}$ for respective incident angles where no polarization dependence exists, and it was further normalized such that the intensity at $\theta=54.7^{\circ}$ are $1 / 3$. 

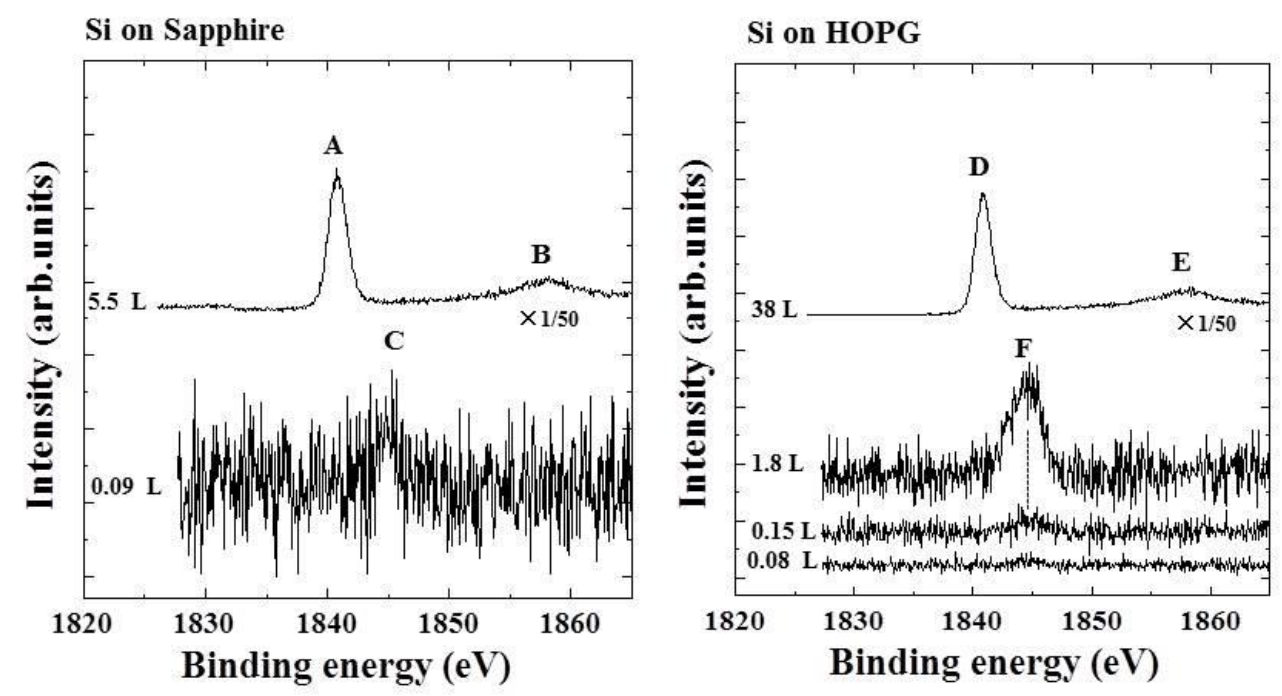

Fig. 1 


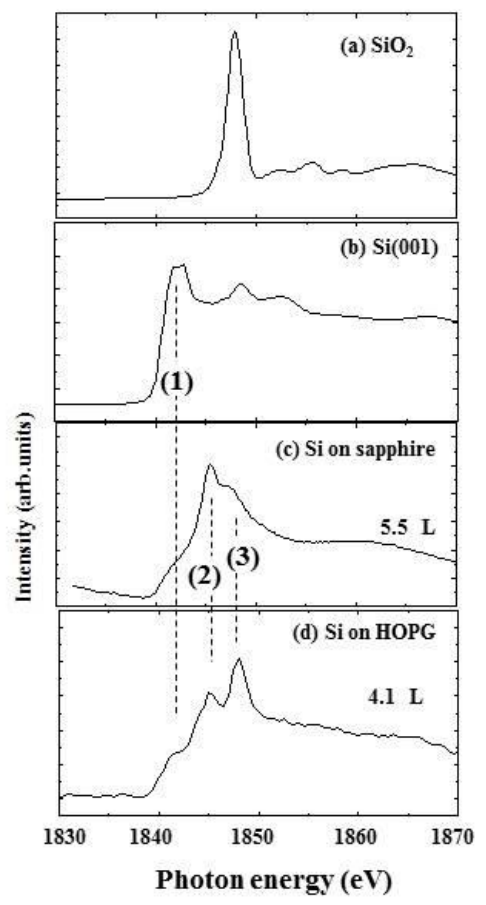

Fig. 2 


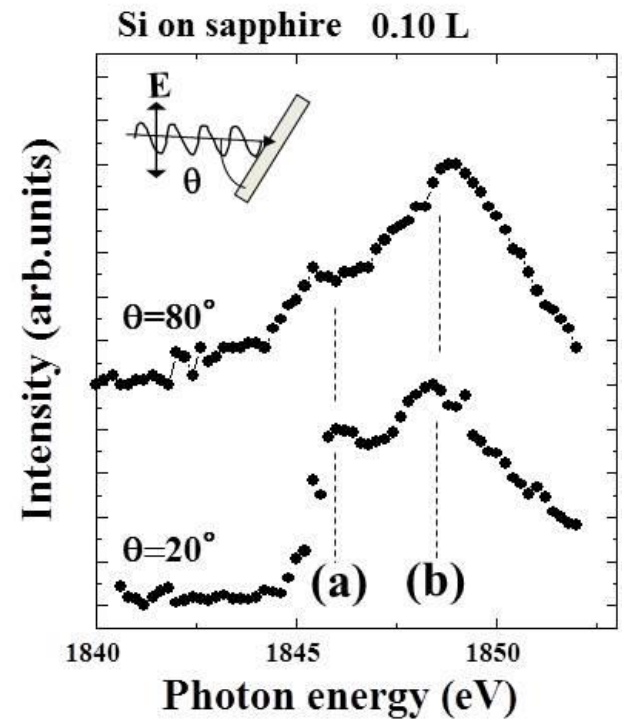

Fig. 3 


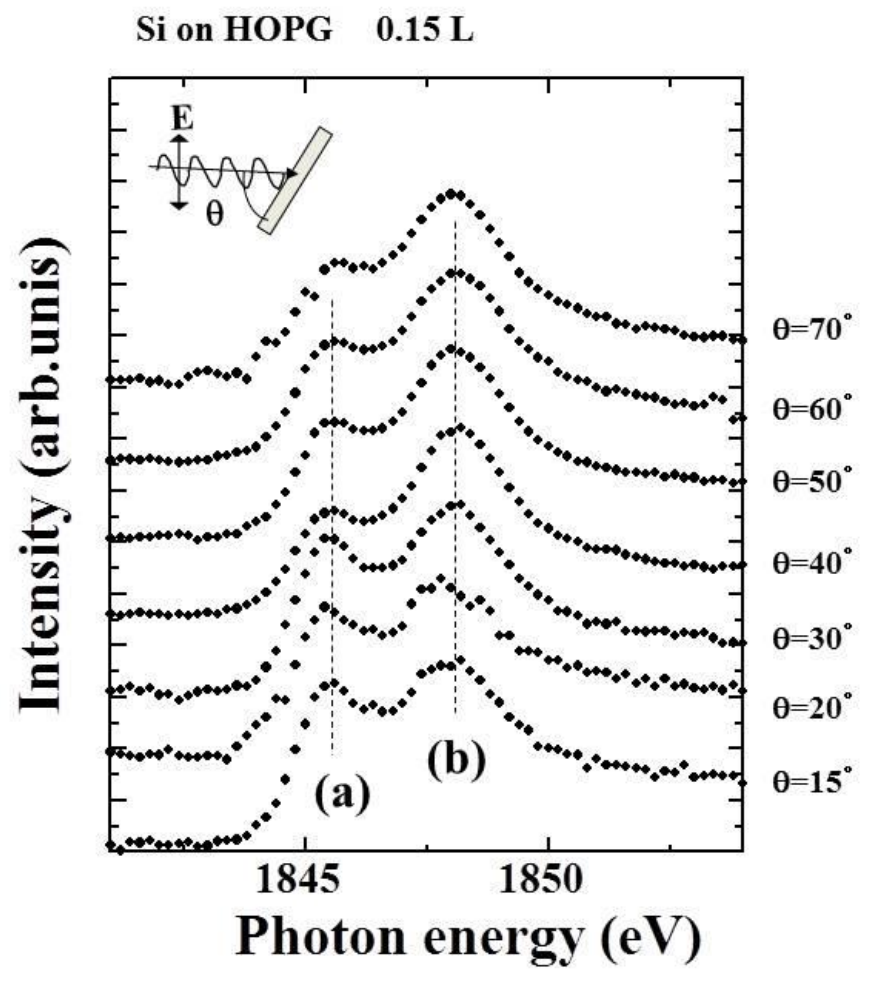

Fig. 4 

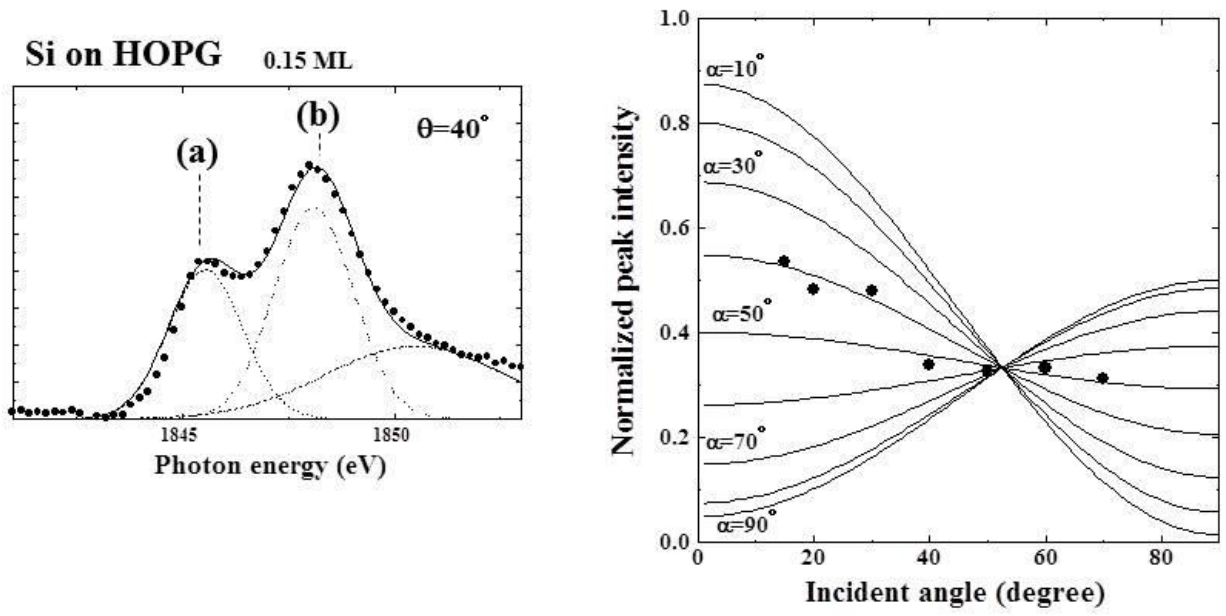

Fig. 5 